\section{Fab libraries for antibody discovery}

\author{
By Kai-Jye Lou, Staff Writer
}

Researchers at Fabrus LLC and The Scripps Research Institute have built a new type of antibody fragment library in which each Fab can be independently assayed via high throughput screening. The company expects the technology will help identify antibody candidates with targets and mechanisms seldom found using conventional antibody discovery technologies. ${ }^{1}$

Fabrus is now building larger Fab libraries and integrating them into its biologics discovery efforts.

Leads for all marketed antibody therapeutics to date were discovered in vivo by immunizing animals with an antigen of interest and then creating hybridomas or isolating B cells that produce antibodies against the antigen.

Current approaches for discovering and optimizing antibodies in vitro rely on libraries generated from display-based systems, in which potential candidates are presented on the surface of cells, phages or ribosomes.

Candidates isolated from both in vitro and in vivo approaches are selected from a pool based on their binding affinity for an antigen of interest.

Affinity-based selection does a good job at finding antagonists of a target but is generally unable to pick out candidates that could have other potentially desirable mechanisms of action like agonism, partial agonism, partial antagonism and allosteric modulation.

Display-based methods also have difficulty generating antibodies against certain classes of molecules like $\mathrm{G}$ protein-coupled receptors and ion channels. Moreover, conventional antibody libraries are not readily compatible with existing high throughput screening technologies such as those used to discover small molecules.

To address these issues, a group led by Fabrus founder and interim CEO Vaughn Smider developed an approach to building arrayed libraries of human Fabs, the antigen-binding fragments of an antibody (see Figure 1, "Building Fab libraries for antibody discovery and optimization"). Each member of the library is placed at a discrete location on a microarray, which allows the Fabs to be assayed independently from one another.

Because each member in the Fab library is evaluated in a separate reaction chamber, there is no direct competition with other members in the library. This is in contrast to affinity-based selection, in which weaker hits could be missed due to competition from stronger hits.

Smider's group screened a library of 10,024 Fabs against a set of 9 target antigens in parallel and identified 85 hits that were able to bind an antigen from the set. The group did not find hits against two of the nine antigens.

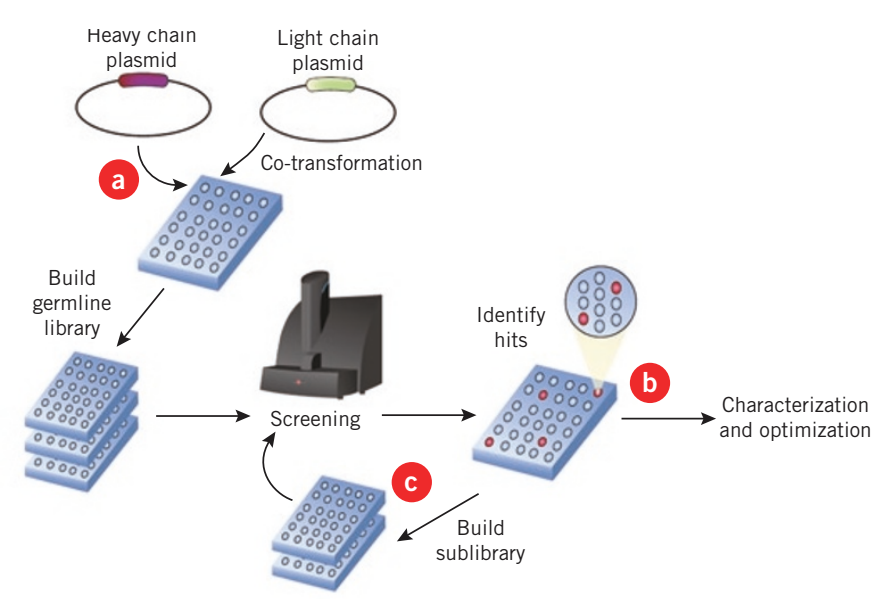

Figure 1. Building Fab libraries for antibody discovery and optimization. Conventional approaches for discovering antibodies rely on either animal immunization with a target antigen followed by the isolation of antibody-producing cells or displaybased systems. In general, such approaches are not readily compatible with the existing high throughput screening methods used to discover small molecule lead candidates.

In Nature Biotechnology, researchers at Fabrus LLC and The Scripps Research Institute report that they have developed an approach to build antibody Fab libraries that can be screened with existing high throughput screening methodologies.

To build these libraries, researchers first co-transform Escherichia coli in wells with plasmids carrying recombined genetic sequences coding for antibody heavy and light chains. Plasmids carrying different heavy chain sequences are co-transformed with multiple light chain plasmids in parallel [a].

The Fabs are obtained after loading the transformed cells into an automated protein expression and purification system.

To screen for hits, researchers load each Fab into another well plate in which each individual well has an array containing multiple antigens of interest [b]. These well plates allow for the screening of individual Fabs against multiple antigens in parallel.

Hits against each antigen can then be characterized and optimized or used to build a sublibrary that is then sent through successive rounds of screening [c].

Importantly, one of the optimized Fabs against human delta-like ligand 4 (DLL4) had partial antagonist activity-a trait that is seldom found in candidates isolated via selection.

Results were published in Nature Biotechnology.

"Our method offers the potential to discover antibodies that wouldn't be easily discovered via conventional approaches," said Smider, who is also an assistant professor of molecular biology at Scripps and corresponding author on the paper. "These protein libraries could facilitate the discovery of antibodies with novel activities or antibodies against targets that are traditionally difficult to target with antibodies." 
Smider added that the Fab libraries are screened in a manner similar to that used for small molecule libraries and could be plugged into existing high throughput screening platforms available at several companies.

"The researchers have done a good job showing that their method could be an alternative to selection-based techniques for antibody discovery," said Sachdev Sidhu, an associate professor of molecular genetics at the University of Toronto. "I was surprised that they were able to identify hits against the set of target antigens using such a small library. Display methods can require libraries with tens of millions of members."

"The classical phage display selection process, which has washing steps, favors the higher affinity binders, but higher affinity binders are not necessarily the best candidates," noted Philippe Mondon, CTO and head of antibody engineering at Millegen. "This library could be a good solution for finding antibodies that do not need to have high affinity, such as those that agonize target function."

\section{"I would see our technology as a way to identify candidates that would be missed by the biological selection approach." \\ - Vaughn Smider, Fabrus LLC}

Smider added that although their method would be more costly than phage display, it would still be considerably cheaper than building a combinatorial chemistry library. "It costs about $\$ 15$ per well using our method, but for combinatorial chemistry libraries, it can cost $\$ 300$ to $\$ 400$ per well.”

Millegen's Mondon thinks that integrating the Fab library into existing antibody discovery platforms will be difficult because the automation systems, such as that used in protein expression and purification, are very specific. He also noted that it will be necessary to integrate the approach with an efficient antibody maturation platform.

As a next step, Sidhu said it will be important for Fabrus to clearly show that its approach can discover candidates with properties not found using existing platforms.

"While it is theoretically possible to develop more complicated screens to identify

Smider agreed that the ability to identify lower affinity antibodies is a potential advantage. "Display technologies rely on biological selection, where the antibodies in a pool that bind best to a particular antigen are the ones that are selected for," said Smider. "We don't know the false negative rates in biological selection experiments, as the antibodies are competing with one another to bind to the target antigen. I would see our technology as a way to identify candidates that would be missed by the biological selection approach."

Indeed, the hits identified in the Nature Biotechnology paper had a wide range of binding affinities.

The new libraries also are able to offer functional screening. "For example," said Smider, "you could carry out an apoptosis functional screen to identify antibodies that induce apoptosis in cancer cells. It is difficult to move directly into a functional screen with display technologies in the absence of some preselection event."

Finally, Smider said his group's approach is amenable to multiplexed discovery, in which members of the Fab library are screened against multiple antigens in parallel. "We showed proof of concept using nine antigens, but we could conceivably scale this up to hundreds or thousands of antigens," he told SciBX.

Despite the potential advantages of the antibody discovery approach described in the Nature Biotechnology paper, Sidhu suggested the amount of time and resources needed to build these libraries could limit their broad application to antibody discovery.

"I think Fabrus would be able to get a lot of mileage out of these libraries for their own programs," he said. "However, it would take a lot of effort to build these protein libraries, and I don't think many labs would be able to use this method to discover antibodies unless they fully commit themselves to using these libraries."

Smider agreed that building large Fab libraries would require a significant investment but noted that academic labs should have the resources to build such libraries on a smaller scale.

"To build these libraries at the scale we're doing it at, with the amount of protein that we're producing, would be a resource-intensive process," he told SciBX. "However, it should be possible to build these libraries on a smaller scale and at a cost that would be reasonable to an academic lab." candidates with more unique properties like agonism, they have yet to show this," he noted. "I think this approach could become an important technology if they are able to show that it can do what current antibody platforms are not able to do."

Millegen uses its directed molecular evolution platform and high throughput functional screening to discover and engineer human antibodies for therapeutic and diagnostic use.

\section{Going bigger}

Smider said his group is now building larger Fab libraries, which will be integrated into Fabrus' discovery efforts. He said the company will initially focus on oncology targets and could have a candidate ready for clinical testing in two to three years.

"While a library size of 10,000 is good enough to find hits against many targets, it could be too small to be optimal and we want to go up to libraries with hundreds of thousands of antibodies," Smider told SciBX. "We are also interested in running functional screens that could be used to identify antibodies that target cancer stem cells and antibodies that could be good candidates for building antibody-drug conjugates."

This week, Fabrus and protein optimization company Ambrx Inc. announced a joint research program to discover antibodies with properties that are optimized for use as antibody drug conjugates.

Fabrus has filed multiple patents covering the methods, protein libraries and hits from the screening studies. The technology and identified hits are available for licensing.

Smider said the company's antibody technology could circumvent royalty-stacking issues associated with conventional antibody discovery platforms.

"Most antibody engineering projects require some sort of display technology, like phage, ribosome or yeast display," he said. "There are a web of patents claiming various aspects of these display compositions and methods. The Fabrus spatially addressed libraries do not use display, nor hybridoma methods, so it is completely outside of this IP."

Fabrus' investors include Pfizer Inc., Opko Health Inc. and multiple angel investors. 


\section{ANALYSIS}

Lou, K.-J. SciBX 3(44); doi:10.1038/scibx.2010.1314

Published online Nov. 11, 2010

\section{REFERENCES}

1. Mao, H. et al. Nat. Biotechnol.; published online Oct. 24, 2010; doi:10.1038/nbt.1694

Contact: Vaughn V. Smider, The Scripps Research Institute, La Jolla, Calif. e-mail: vvsmider@scripps.edu
COMPANIES AND INSTITUTIONS MENTIONED

Ambrx Inc., La Jolla, Calif.

Fabrus LLC, La Jolla, Calif.

Millegen, Labege, France

Opko Health Inc. (NYSE-A:OPK), Miami, Fla.

Pfizer Inc. (NYSE:PFE), New York, N.Y.

The Scripps Research Institute, La Jolla, Calif.

University of Toronto, Toronto, Ontario, Canada 